\title{
Determining Role of the Optic Vesicle in Orbital and Periocular Development and Placement
}

\author{
KENNETH LYONS JONES, ${ }^{(1) i}$ MARILYN C. HIGGINBOTTOM, AND DAVID W. SMITH \\ Department of Pediatrics, University of California, San Diego School of Medicine, La Jolla. California 92093 /K. L. \\ J., M. C. H.J, and Dysmorphology Unit, Department of Pediatrics, University of Washington School of Medicine. \\ Seattle. Washington, USA [D. W. S.]
}

\section{Summary}

Nine patients with aberrations in development and placement of the eyes and periocular structures who also had serious defects in central nervous system development were evaluated in order to better understand normal ocular development. Included were an incompletely developed twin stillborn infant who lacked both eyes and the nose, a stillborn infant with cyclopia hypognathia, 6 spontaneous abortuses with varying degrees of holoprosencephaly, and a 17-year-old male with a serious defect in central nervous system development whose right eye was positioned laterally above the right ear. In all cases, evidence indicates that orbital and
Data from both animal and human studies have suggested that these structures are to a great extent independently derived $(3,6)$. The purpose of this report is to present evidence from nine patients with aberrations in development and placement of their eyes that orbital and periocular structures are determined by the optic vesicle, a neural organ which is an outpouching of the forebrain.

\section{PATIENTS AND METHODS OF INVESTIGATION}

Details relative to development of the ocular, orbital, and periocular structures are presented below and are documented in Figures 1 to 8.

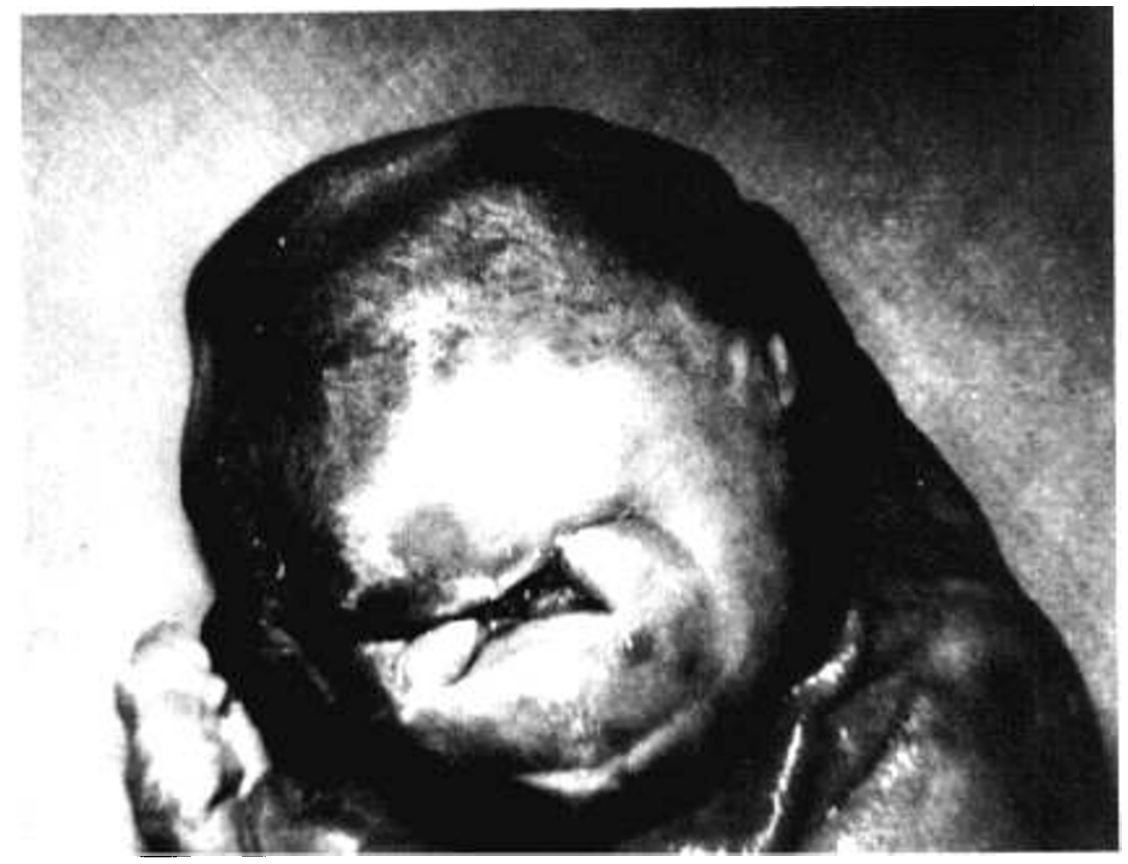

Fig. 1. Patient 1. Lack of both eyes and the nose. The mouth is represented by an irregular transverse cleft.

periocular structures are determined by the underlying optic vesicle rather than independently derived as has been suggested by previous studies.

\section{Speculation}

The intimate developmental relationship between the forebrain and structures of the upper face suggests that alterations of periocular and bony orbital structure and placement may well reflect defects in underlying brain development.

The developmental relationship between the eye, the periocular structures, and the bony orbit has not been completely understood.

\section{PATIENT 1 (FIG. 1)}

This stillborn female twin was delivered after a gestation of 22 weeks. The face of the child was not developed. Both eyes and the nose were absent. The mouth was represented by an irregular transverse cleft. There were two small skin tags measuring 4 and $3 \mathrm{~mm}$ on the left and right sides of the head, respectively, presumably denoting the external ears. Histologic evaluation of sections through the central portions of the face revealed only small clusters of disorganized retinal tissue with no additional identifiable ocular, bony orbital, or periocular structures. Examination of the brain demonstrated continuity of the frontal lobes in the midline with absence of the falx and the olfactory bulbs. There was a 


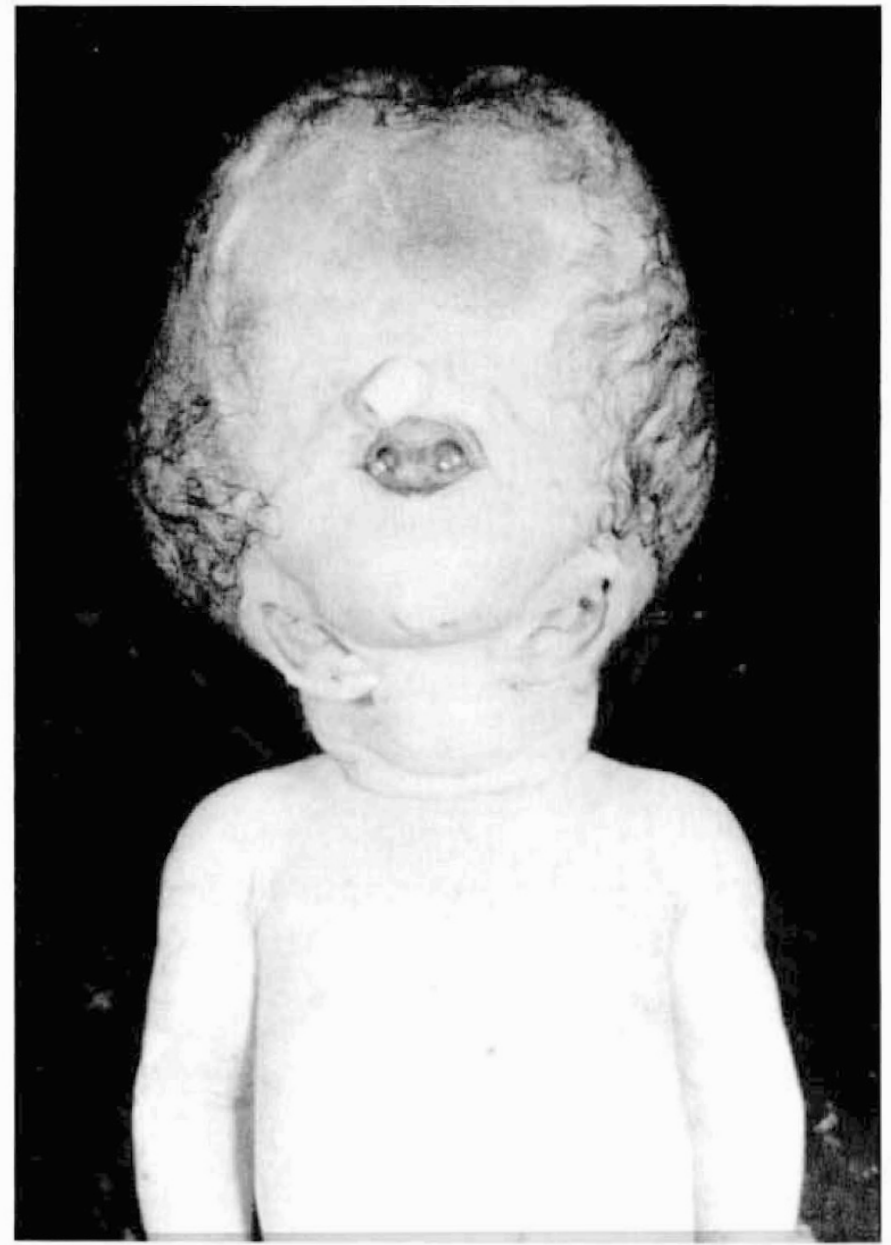

Fig. 2. Patient 2. A stillborn infant with cyclopia hypognathia. Fused eyes within a single orbit and midline proboscis.

single horseshoe-shaped ventricular cavity. The corpus callosum and the septum pellucidum were absent. Radiographs confirmed the lack of bony orbital structures.

PATIENT 2 (FIG. 2)

This stillborn male infant was delivered after a gestation of 40 weeks. The two eyes were fused and contained within a single midline bony orbit. The palpebral fissure was represented by an elliptical opening measuring $28 \mathrm{~mm}$ in the horizontal and 15.5 $\mathrm{mm}$ in the vertical axis. The upper and lower lids each consisted of a single arched fold of skin. Neither lid possessed a tarsal plate, but each had a row of lashes along the outer margin. No supraorbital ridge was evident, and there were no eyebrows. The altered positions of the extrinsic ocular muscles are depicted in Figure 3. The left cornea was larger than the right. The two optic nerves were fused into a single midline structure directed posteriorly from the globe at the point where the two eyes were fused. Radiographs of the skull demonstrated a single midline bony orbit and a single optic foramen (Fig. 4). Computerized axial tomograms confirmed the presence of a single orbit and outlined the course of the single, centrally located optic nerve. The forebrain was comprised of a holosphere which lacked dividing structures and olfactory bulbs. There was a large median dorsal cyst lying between the holosphere and the cerebellum.

\section{PATIENTS 3 THROUGH 8 (FIGS. 5 AND 6)}

These specimens which represent six spontaneous abortuses with varying degrees of holoprosencephaly were kindly provided for the study by Dr. Hideo Nishimura and Dr. Takahasi Tana-

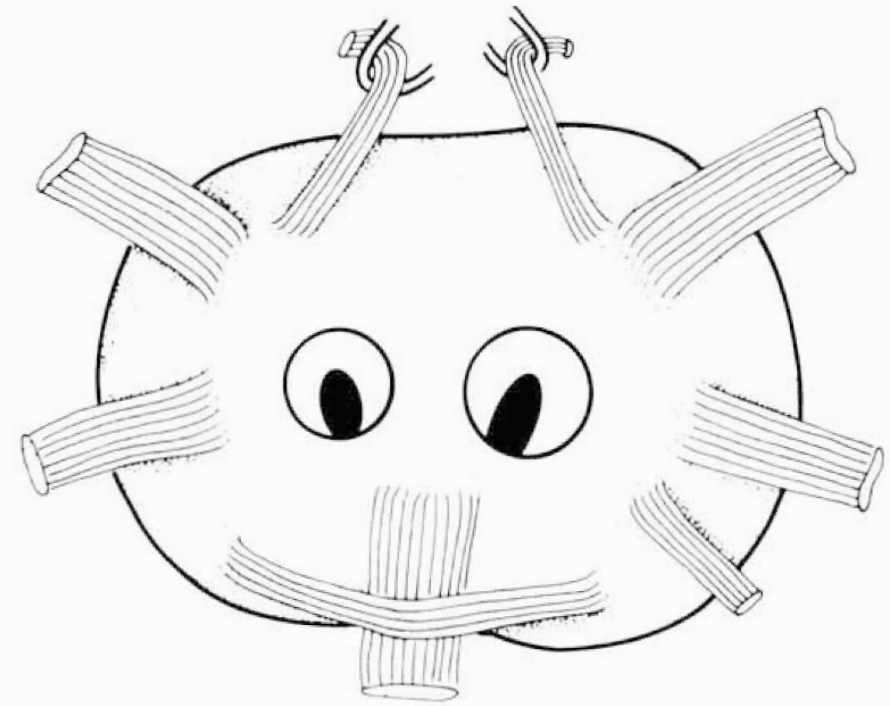

Fig. 3. Anterior view of the extrinsic ocular muscles of patient 2. Ten muscle bands are attached to the fused eyes. The inferiorly placed midline muscle band presumably consists of the medial recti and the right inferior rectus. The remainder of the extrinsic ocular muscles are abnormally placed and represented as discrete bands.

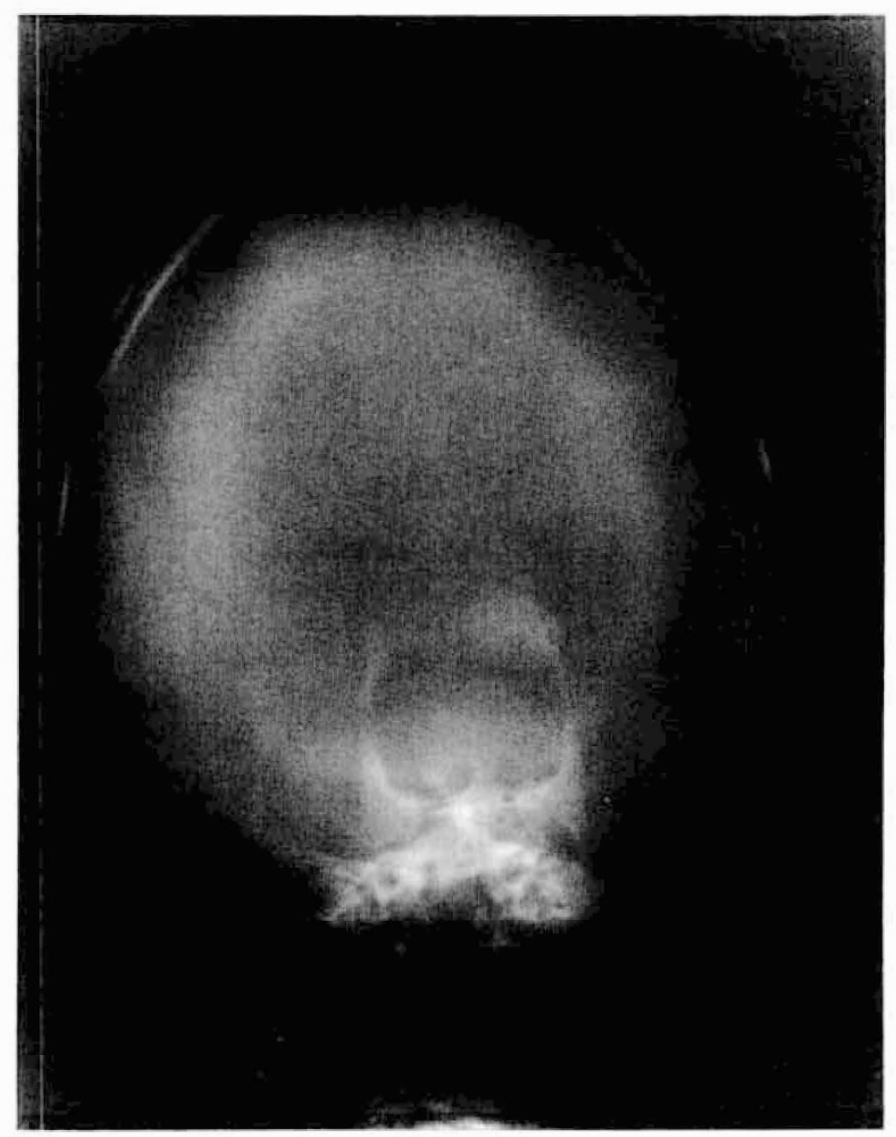

Fig. 4. Anteroposterior radiograph of the skull of patient 2. Single midline bony orbit and single optic foramen.

mura of Kyoto University. Japan. The earliest is an II-mm crown/ rump length human embryo ( 38 days). The other five include one embryo of $17 \mathrm{~mm}$ ( 43 days). two of $18 \mathrm{~mm}$ (47 days), one of 22 $\mathrm{mm}$ ( 51 days), and one of $26 \mathrm{~mm}$ ( 56 days). In all cases, histologic evaluation revealed early periocular condensation in proximity to the eyes. 

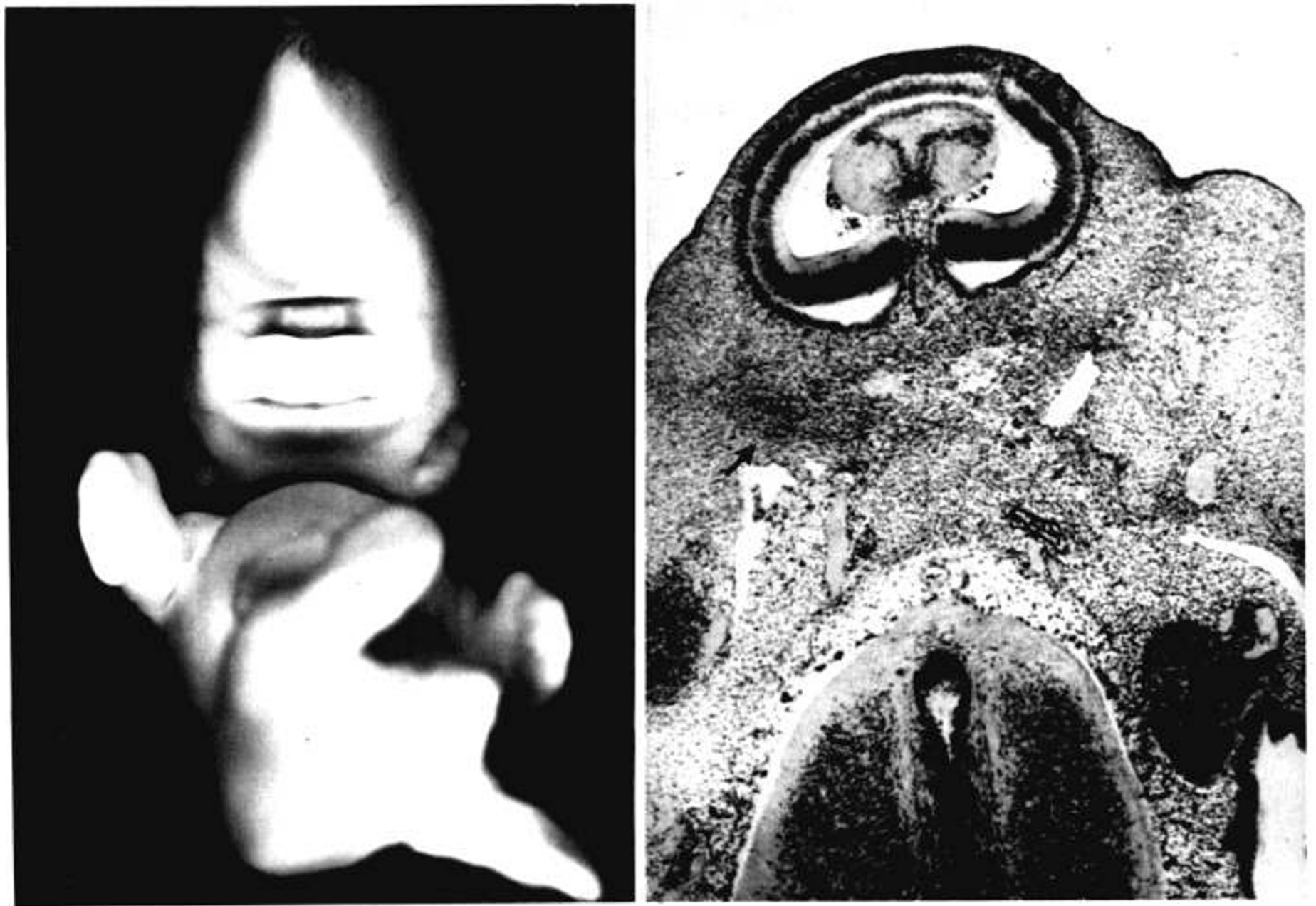

Fig. 5. A $17-\mathrm{mm}$ crown/rump length (43 days) human embryo with holoprosencephaly. On the microscopic section, the arrow points to darkly staining cells representing mesenchymal condensations which are in proximity to the fused eyes.

\section{PATIENT 9 (FIG. 7)}

This patient, previously reported by Peterson et al. (7), is a 17year-old white male whose structural defects are confined to the craniofacial area. All of the defects were present at birth. The left side of the face is clinically normal; however, the structures of the right side are markedly distorted. The right eye is positioned above the right ear on the lateral aspect of the head. The globe is not palpable; however, a palpebral fissure is represented by a round opening measuring $2 \mathrm{~mm}$ in diameter. A clump of eyelashes protrudes from the palpebral fissure. Contraction of the area directly surrounding the opening occurs when the clinically normal left palpebral fissure is forcefully and voluntarily closed. Tears flow from the opening. The eyebrow conforms in position to the superior rim of a small bony orbit. The anterior calvaria is markedly asymmetric as a result of bony overgrowth of the right forehead and hypoplasia of the right maxilla. The right nostril is absent; however, there is a $5-\times 6-\mathrm{mm}$ elevated skin tag $2 \mathrm{~cm}$ superior to the corner of the mouth on the right. There is a line of hair, similar to an eyebrow which conforms in position to a bony ridge and marks the inferior extension of the forehead on the right side. Radiographs of the skull demonstrate bony overgrowth of the right forehead, slight lateral displacement of the left orbit and a small right orbit on the side of the head conforming in position to the rudimentary ocular structures. A computerized axial tomogram of the head reveals an asymmetric skull involving primarily the frontal, sphenoidal, and ethmoidal complex. Underlying the left orbit, there is a large sphenoidalethmoidal, air-filled sinus. Within the small right orbit, which is eccentrically placed, there is an abnormal globe (Fig. 8). The osseous anomaly is accompanied by a brain parenchymal defect characterized by an asymmetric ventricular system and a large, low-density image in the right hemisphere suggestive of schizencephaly.

\section{DISCUSSION}

The combined observations in these nine patients suggest that the periocular and bony orbital structures depend for their normal development on the early position and development of the underlying optic vesicle. Evidence supportive of this conclusion is as follows.

In patient 1, who had a serious defect in brain development associated with a complete lack of normal ocular structures both grossly and microscopically, there was a corresponding lack of any bony orbital or periocular structures. Cesare Taruffi, in his classic "Storia della Teratologia" published in 1891 (12), reviewed cases of 7 additional patients with similar alterations in development of the face and brain. He was the first to suggest that the facial abnormalities, including the absence of orbital and periocular structures, were secondary to aplasia of the anterior and medial brain.

In patient 2, who had fused eyes and a single fused optic nerve, there was a single bony orbit, a single optic foramen, and a single palpebral fissure. In addition, there was marked alteration in the position, origin, and insertion of the extrinsic ocular muscles. The abnormal position of the muscles conformed to the structure of both the eyes and the single bony orbit. This is consistent with the hypothesis that the position of the extrinsic ocular muscles is determined by and dependent upon the developmental relationship between the optic vesicle and adjacent mesoderm. Because 

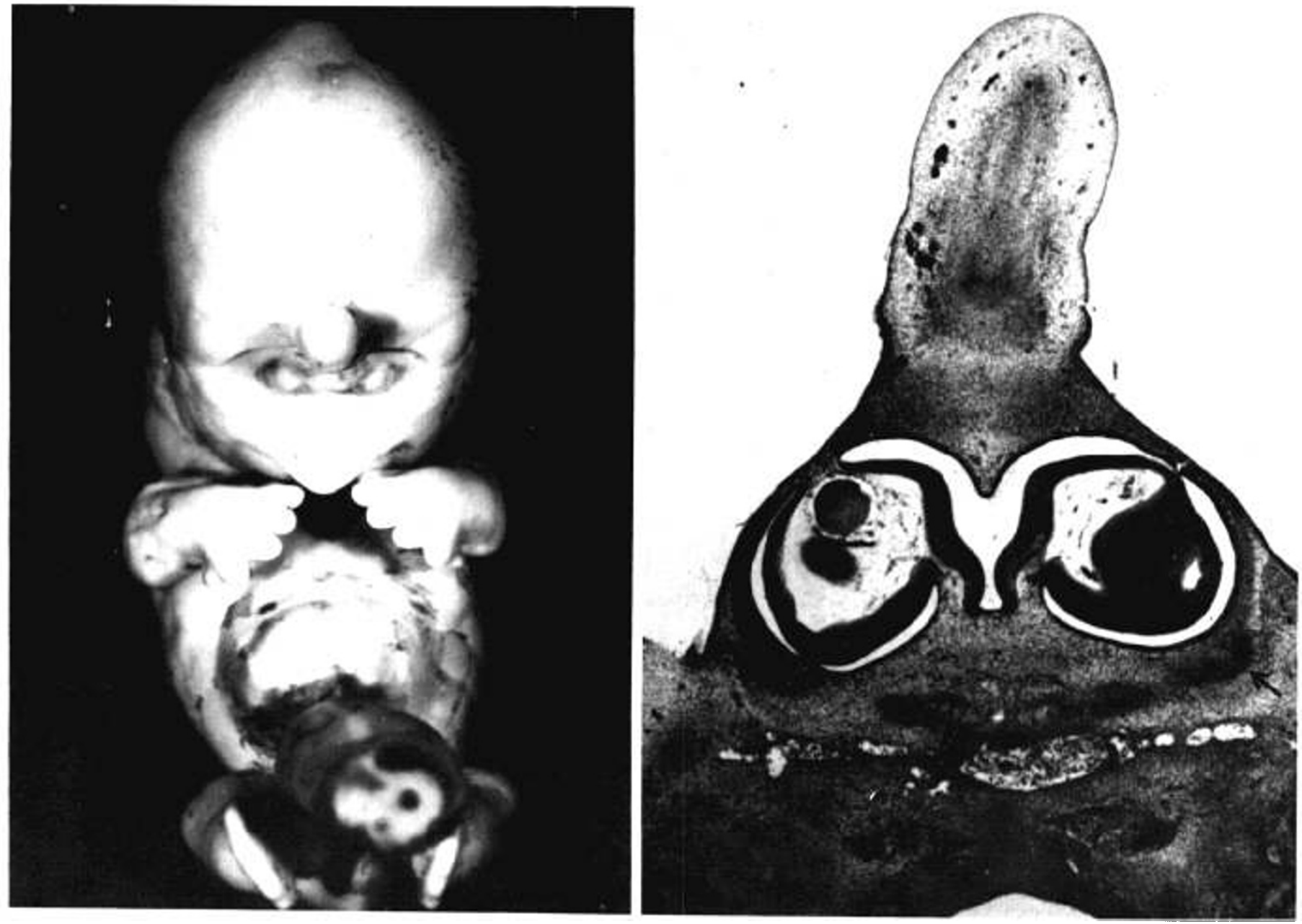

Fig. 6. A 22-mm crown/rump length (51 days) human embryo with holoprosencephaly. On the microscopic section, the arrow marks darkly staining cells which represent mesenchymal condensations surrounding the partially fused eyes.

there was no medial orbital wall, the inferior oblique muscles. which normally attach to the outer medial rim of the floor of the bony orbit, had no place to attach, and in this case, were displaced onto a midline mass of muscle connected to the inferior aspect of the globe (Fig. 3). This mass of muscle is believed to consist of the medial recti and the right inferior rectus. The former were displaced inferiorly because of lack of medial eyeball structures on which to insert.

The extrinsic ocular muscles have been dissected in at least nine additional patients with varying degrees of holoprosencephaly (4. 13). Although there has been marked inconsistency in the placement of the individual muscles from patient to patient, it is interesting to note that, in general, a situation similar to that seen in patient 2 has been observed. The muscles innervated by cranial nerve III tend to be fused (superior, medial, and inferior recti and inferior oblique), whereas those innervated by cranial nerves IV and VI tend to be separate (superior oblique and lateral rectus. respectively).

Neurohistologic evaluation of the six spontaneous abortuses with varying degrees of holoprosencephaly, patients 3 through 8 , gives further evidence that orbital and periocular structures are determined by the development of the optic vesicles. In each of these specimens, an early mesenchymal condensation, the forerunner of the bony orbit and extrinsic ocular muscles, was noted in proximity to the altered position of the developing ocular tissues rather than more laterally as would be anticipated if the mesodermally derived orbital and periocular structures developed independently.

In patient 9, the orbital and periocular structures on the right side were above the right ear. The palpebral fissure and bony orbit were small and conformed to the ectopic position of the globe. Ability to contract the area immediately surrounding the rudimentary right eye indicates that the orbicularis oculi muscle and its motor innervation are also determined by the position of the optic vesicle.

The interpretation that the orbital and periocular structures depend on the underlying optic vesicle for normal development and position contradicts the theory set forth by both Mann (6) and Duke-Elder (3), contending that the bony orbit and periocular structures are for the most part self-determined. Evidence for that theory was drawn primarily from evaluation of patients with clinical anophthalmia who had relatively normal periocular and bony orbital structures. However, the majority of cases used to support the hypothesis of independent development did not include microscopic examination of the orbits, and microphthalmia was not excluded. Of those cases in which anophthalmia was demonstrated histologically, evaluation of at least two indicated that the optic vesicle had initially formed and, therefore, could have initiated development of the periocular and bony orbital structures $(8,9)$.

Extirpation and grafting experiments on the eye of chick embryos in addition to studies of an anophthalmic strain of mice have also been used to illustrate the independent development of the orbital and periocular structures $(10,11)$. In the extirpation experiments, either one or both primary optic vesicles were removed at the 10 to 12 somite stages. Examination of the embryos at a later stage of development revealed that the orbits and certain other juxtaorbital structures were reduced in size but were clearly present. Although that study has been used to support the concept of independent development, the presence of the primary optic 

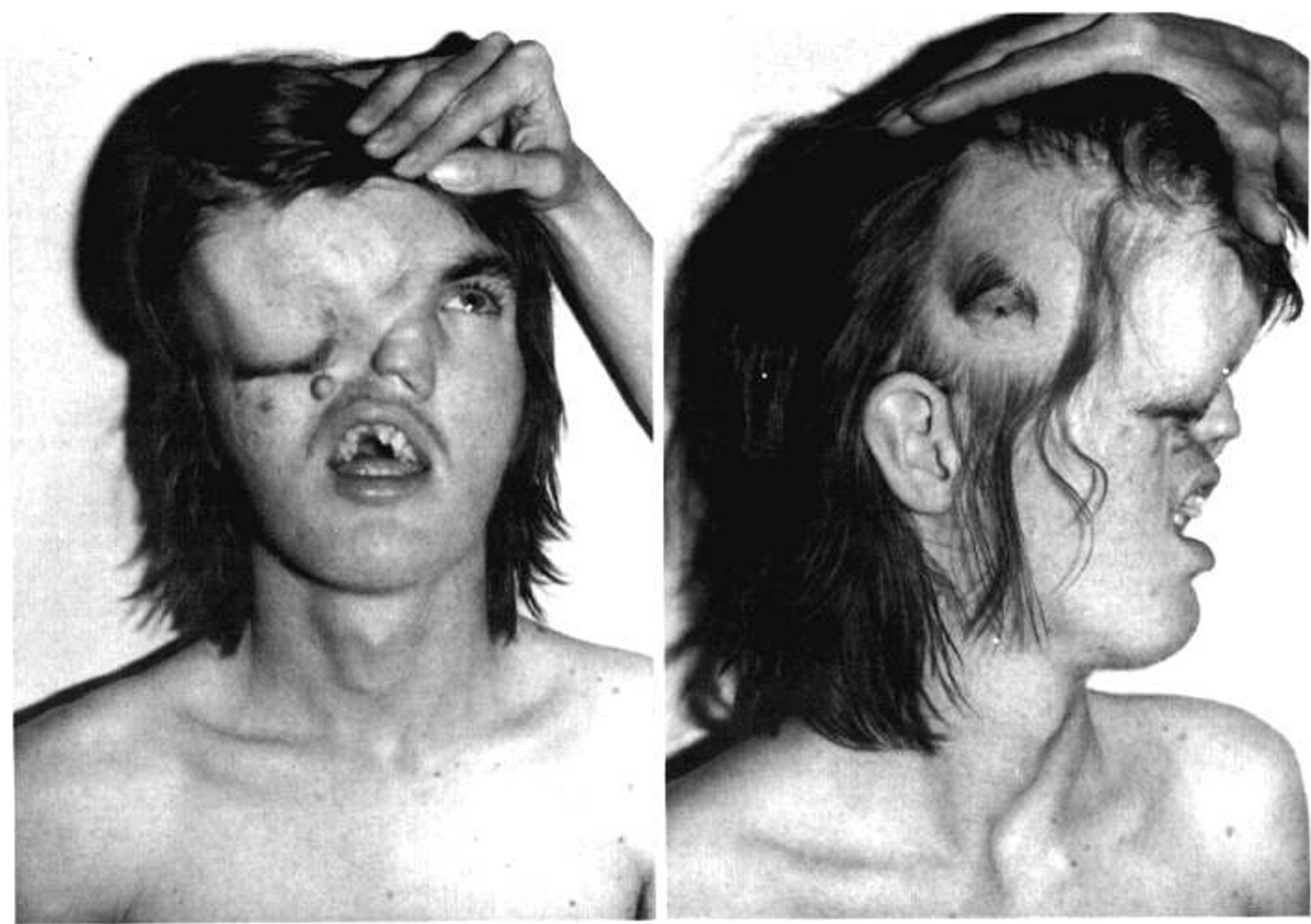

Fig. 7. Patient 9. The right eye is positioned above the right ear. The palpebral fissure on that side is short with eyelashes protruding from it. Above it. there is an eyebrow conforming in position to the superior rim of a small bony orbit. The forehead on the right protrudes anteriorly, the right nostril is absent. and there is a line of hair similar to an eyebrow which marks the inferior extension of the forehead on the right side.

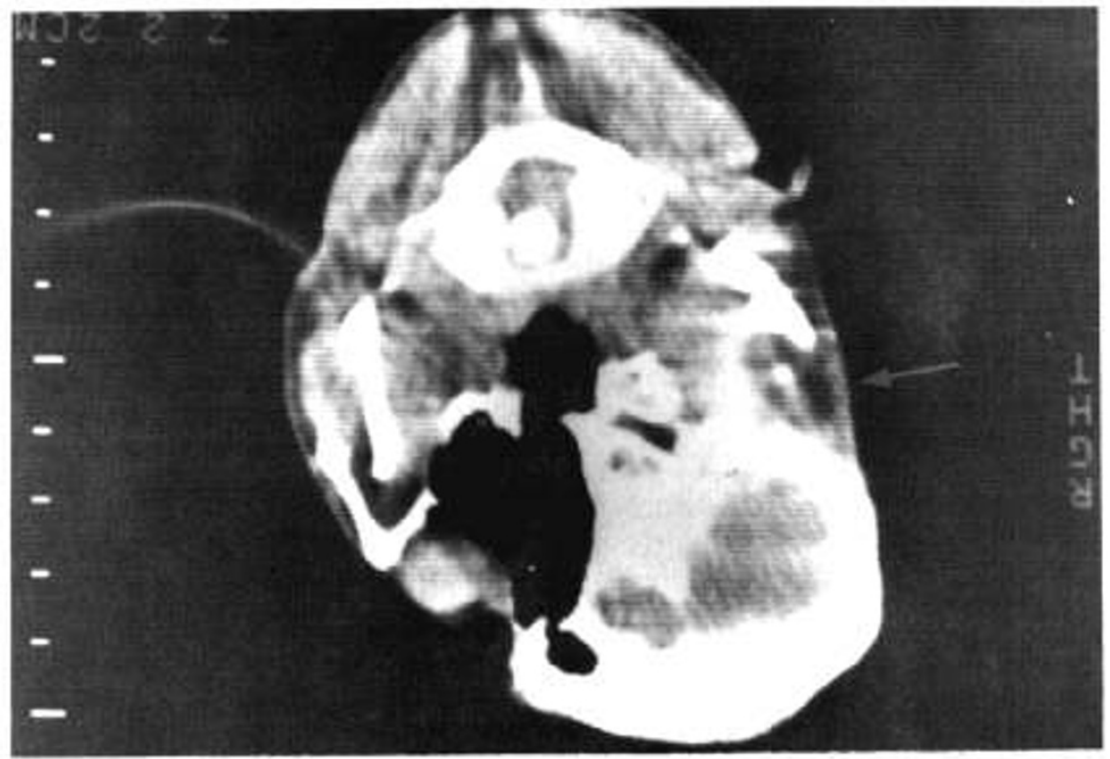

1:ig. 8. Computerized axial tomography from patient 9. Arrow, small laterally placed right bony orbit. Note within it the abnormal globe. The asymmetry of the anterior calvaria is due to bony overgrowth in that area.

vesicle early in development most probably provided the stimulus for subsequent differentiation of orbital and periocular structures. In the study of an anophthalmic strain of mice, serial histologic studies at various stages of development demonstrate the presence of ocular structures in the mice as late as 13 days gestation, a time when periocular mesodermal condensation has already commenced (1). This suggests that the development and position of the orbital and periocular structures in those animals noted to be 
anophthalmic at the time of birth were determined by ocular structures that were present when mesenchymal condensation began.

Appreciation that normal development and position of the orbital and periocular structures depends upon the position and development of the optic outpouching of the forebrain has potential clinical value because alterations in these structures are readily apparent on the physical examination. The following clinically relevant conclusions seem warranted: (1) the existence of periocular and bony orbital structures implies that the optic vesicle was present at an early stage in development. Clinical anophthalmia in such situations is therefore the result of degeneration rather than aplasia of the optic vesicle; (2) ocular hypotelorism should suggest a defect in formation of the forebrain as has been previously proposed by DeMyer et al. (2); (3) palpebral fissure length relates to eye size. Short palpebral fissures, as seen in disorders such as the fetal alcohol syndrome 5 , are secondary to decreased growth of the eye; (4) because the optic vesicle plays a determining role in the formation of the periocular and bony orbital structures. any alteration in the latter may be a clue to a more basic defect in the underlying eye.

\section{REFERENCES AND NOTES}

I. Chernoff, G. F., and Jones, K. L.: (unpublished data)

2. De Meyer, W.. Zeman, W.. and Palmer, C. G. L.: The face predicts the brain diagnostic significance of median facial anomalies for holoprosencephaly (arhinencephaly). Pediatrics, 34: 256 (1964).

3. Duke-Elder, S.: Normal and Abnormal Development. In: System of Ophthalmology. Vol. 3 (C. V. Mosby Co., St. Louis, 1964).

4. Humphrey. R. R.: A case of cyclopia in homo. Anat Rec., 28: 207 (1924).

5. Jones, K. L., Smith, D. W., Ulleland, C. N., and Streissguth, A. P.: Pattern of malformation in offspring of chronic alcoholic mothers. Lancet, I: 1267 (1973).

6. Mann. 1.: Developmental Abnormalities of the Eye. (Cambridge University Press.
London, 1937).

7. Peterson, M. Q., Cohen. M. M., Jr., Sedano, H. O., and Frerichs, C. T.: Comments on frontonasal dysplasia. ocular hypertelorism and dystopica canthorum. Birth Defects (original article series). 7: 120 (1971).

8. Redslop. E.: Anophtalmie vraie et anophtalmie apparente chez un nouveau-ne Ann. Ocul., 169: 433 (1932).

9. Sedan, J.: L'anophtalmie de pores nephro-ectopiques. Ann. Ocul., 189: 392 (1956).

10. Silver, J., and Hughes. A. F. W.: The relationship between morphogenetic cell death and the development of congenital anophthalmia. J. Comp. Neurol.. 1.57: 281 (1974).

11. Silver, P. H. S.: In ovo experiments concerning the eye, the orbit and certain juxta-orbital structures, in the chick embryo. J. Embryo. Exp. Morphol.. 10: 423 (1962)

12. Taruffi, C.: Storia della Teratologia. Parte Prima. Tomo VI. (Rigia Tipografia. Bologna, 1981).

13. Wilder, H. H.: The morphology of cosmobia: speculations concerning the significance of certain types of monsters, Am. J. Anat., 8: 355 (1908).

14. Consent to photograph was obtained by the authors.

15. We are especially grateful to Dr. Alasdair Hunter. Winnipeg. Manitoba who referred to us the clinical and pathologic data on patient 1. to Dr. Salvatore Stella and Dr. Russell Van Dyke who worked on the dissection of the extrinsic ocular muscles of patient 2. to Dr. Hideo Nishimura and Dr. Takashi Tanimura, Kyoto University, who provided one of us (D. W. S.) the opportunity to evaluate patients 3 through 8: to Dr. Michael Cohen who made us aware of patient 9, and to Dr. Hector James who helped with the neuroradiographic evaluation.

We acknowledge the contributions of Lyle Harrah. Research Librarian. May Ann Harvey. George Rutherford. Vincent Massullo, and Mary Bastian for translation and the contributions of Kathleen Johnson for assistance in preparation of the manuscript.

16. Requests for reprints should be addressed to: Kenneth Lyons Jones, M.D. Department of Pediatrics, University Hospital, H-814-B. 225 W. Dickinson Street, San Diego, CA 92103 (USA)

17. This research was supported in part by the Institute for Pediatric Service of the Johnson and Johnson Baby Products Company.

18. Received for publication August 29.1979.

19. Accepted for publication December 11, 1979. 